\title{
Linx
}

Revue des linguistes de l'université Paris X Nanterre

$81 \mid 2020$

Enseignement des langues : la grammaire et la linguistique sont-elles solubles dans la pédagogie de projet ?

\section{Pour une préparation anticipée des futurs enseignants du secondaire à une approche impliquante de la grammaire}

Preparing future high school English teachers to an engaging approach to

teaching grammar

Hélène Josse de la Gorce et Céline Thurel

\section{(2) OpenEdition}

Journals

Édition électronique

URL : http://journals.openedition.org/linx/6917

DOI : $10.4000 / \operatorname{linx} .6917$

ISSN : 2118-9692

Éditeur

Presses universitaires de Paris Nanterre

Édition imprimée

Date de publication : 15 décembre 2020

ISSN : 0246-8743

Référence électronique

Hélène Josse de la Gorce et Céline Thurel, « Pour une préparation anticipée des futurs enseignants du secondaire à une approche impliquante de la grammaire », Linx [En ligne], 81 | 2020, mis en ligne le 15 décembre 2020, consulté le 29 janvier 2021. URL : http://journals.openedition.org/linx/6917 ; DOI : https://doi.org/10.4000/linx.6917

Ce document a été généré automatiquement le 29 janvier 2021.

Département de Sciences du langage, Université Paris Ouest 


\title{
Pour une préparation anticipée des futurs enseignants du secondaire à une approche impliquante de la grammaire
}

\author{
Preparing future high school English teachers to an engaging approach to \\ teaching grammar
}

Hélène Josse de la Gorce et Céline Thurel

\section{La grammaire de l'anglais à la Sorbonne Nouvelle}

\subsection{Enseignement frontal et transmissif en Licence et en Master1}

1 Les étudiants qui suivent des études d'anglais (Licence LLCER) à la Sorbonne Nouvelle bénéficient de 2 heures hebdomadaires de grammaire/linguistique anglaise sur 25 semaines chaque année. Ces heures sont dispensées en TD, dans des groupes de 30 à 40 étudiants. Pour autant, l'enseignement y est dispensé de manière relativement frontale et transmissive (Therer \& Willemart, 1984) puisque l'enseignant explique le point de grammaire et s'interrompt pour poser quelques questions ou faire corriger des exercices que les étudiants sont censés avoir préparés à la maison. Le programme tente de couvrir la morpho-syntaxe du groupe verbal ( 1 ère année), du groupe nominal ( 2 ème année) et la phrase ( 3 ème année) et permet une ouverture vers des problématiques linguistiques plus larges telles que l'iconicité de la langue, sa linéarité, sa subjectivité ou encore la gestion de l'information. La finalité de ces cours est de faire passer les étudiants d'un statut d'utilisateurs de la langue à un statut d'experts. L'enseignement vise essentiellement un renforcement des savoirs déclaratifs sur le fonctionnement de la langue anglaise, plus qu'une amélioration de la compétence langagière.

2 En 2014/2015, nous avons mené une enquête sur notre cohorte d'étudiants de Licence d'anglais $2^{\text {ème }}$ année $(\mathrm{N}=169)$. Elle nous a permis de poser trois constats : 1) un taux 
d'échec important (35\% pour le cours de grammaire contre $26 \%$ toutes matières confondues) ; 2) un fort absentéisme ; 3) un goût limité pour la linguistique.

3 Ces trois constats cumulés nous ont amenées à questionner le format de ce cours qui plaçait les apprenants dans une posture passive et à initier une recherche-action. Incidemment, le format du cours nous paraissait entrer en contradiction avec la pratique de la grammaire que certains de nos étudiants devront exercer quand ils deviendront enseignants dans le secondaire.

\subsection{Introduction à un enseignement actionnel en Master2 MEEF}

4 Ce type d'enseignement frontal et transmissif se poursuit en 1 ère année de MEEF second degré où l'objectif affiché est la réussite aux concours de recrutement de l'enseignement. Les lauréats se trouvent l'année suivante en position d'enseignement et vont devoir enseigner la grammaire dans le cadre de l'approche actionnelle préconisée dans le secondaire en France suite, entre autres, à la publication du Cadre Européen Commun de Référence pour les Langues (CECRL) en 2001. L'étude de la langue n'y est plus présentée comme une activité à part entière mais comme un des éléments qui peut favoriser le développement de la compétence langagière. Le CECRL s'appuie sur des travaux qui datent des années 1970 et développent l'idée que les langues sont apprises pour communiquer et interagir. Il préconise une approche actionnelle fondée sur des tâches :

Les tâches pédagogiques communicatives (contrairement aux exercices formels hors contexte) visent à impliquer l'apprenant dans une communication réelle, ont un sens (pour l'apprenant), sont pertinentes (ici et maintenant dans la situation formelle d'apprentissage), exigeantes mais faisables (avec un réajustement de l'activité si nécessaire) et ont un résultat identifiable (ainsi que d'autres, moins évidents dans l'immédiat). (CECRL, $2001: 121$ )

Dans ce cadre, Spada et Lightbown (2008) soulignent l'importance de favoriser l'intégration du travail sur la langue à un moment du cours où les apprenants sont impliqués dans des activités dont le focus est le sens et non la forme.

6 Les textes français, et particulièrement le Socle Commun de Connaissances, de Compétences et de Culture (2015), n'interdisent pas l'attention spécifique à la langue mais cette attention doit, là encore, rester au service du sens. C'est ce que certains chercheurs nomment Isolated Form-Focused Instruction. Spada et Lightbown (2008) précisent qu'il s'agit de proposer des activités spécifiquement centrées sur la langue mais qui s'inscrivent dans un enseignement interactionnel.

7 Les enseignant-stagiaires, inscrits en M2 MEEF, sont ainsi confrontés à une double difficulté : enseigner la grammaire dans une approche qu'ils n'ont pas pratiquée à l'université et trouver un équilibre entre attention à la langue pour elle-même et primauté de la langue comme outil d'interaction sociale.

8 A l'INSPE de Paris, les stagiaires bénéficient de 8 heures de formation spécifiquement dédiées à cet apprentissage ; ceux inscrits à la Sorbonne Nouvelle peuvent préférer suivre un séminaire pratique de 24 heures. Notre article de 2015 en détaille le programme et insiste sur la variété des moments grammaticaux qui peuvent être mis en place en cours dans le secondaire. Nous revenons ici sur le moment le plus explicite et qui correspond le plus nettement à la Form-Focused Instruction, celui de la Conceptualisation Inductive Complète (CIC) que nous avons modélisée en cinq étapes : 


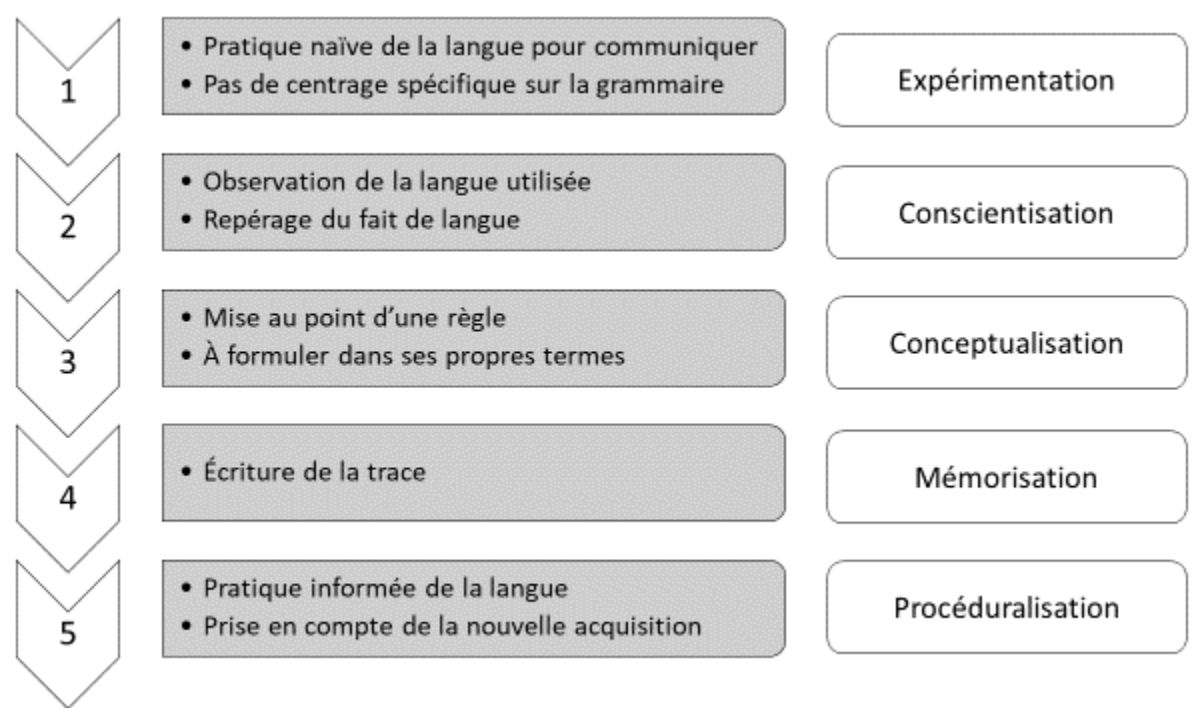

Figure 1 : Les 5 étapes de la conceptualisation inductive complète et les opérations mentales correspondantes - INSPE de Paris et Sorbonne Nouvelle

9 Le cours proposé aux étudiants-stagiaires de M2 MEEF montre comment intégrer la réflexion sur la langue (étapes 2 à 4 ) dans un cours actionnel (étapes 1 et 5). En effet, seules les étapes centrales se concentrent sur la langue de manière explicite et prioritaire. Lors de la phase 1 , on laisse les faits de langue émerger de manière implicite en privilégiant l'intention de communiquer afin que les élèves s'impliquent le plus possible. Dans la phase 5, on peut commencer par une activité qui s'apparente à un exercice afin de finaliser la mémorisation des savoirs déclaratifs. Mais on propose ensuite aux élèves une tâche authentique afin que le fait de langue soit pratiqué dans un contexte signifiant. Ajoutons que le format du cours aux étudiants de M2 MEEF se veut actionnel puisque les stagiaires sont mis en situation de construire des séances incluant une CIC et de produire des activités et des tâches incluant le travail sur des faits de langue.

\subsection{Difficultés ressenties par les étudiants de Master2 MEEF}

Afin d'aider les stagiaires dans leur apprentissage de l'enseignement actionnel de la grammaire, nous leur avons proposé d'entamer une démarche réflexive (Berthiaume \& Rege, 2013) sur leur rapport à la grammaire. Nous sommes parties d'un questionnaire réflexif administré 3 semaines après la rentrée des classes et d'un autre 5 mois après la rentrée.

11 Pour le questionnaire initial, à l'affirmation «J'estime qu'il faut faire de la grammaire en classe », 63 des 65 répondants ont répondu positivement, montrant là une grande homogénéité. A l'affirmation «Je pense avoir bien enseigné la grammaire depuis la rentrée », 25 répondent « oui, vraiment » ou « oui, plutôt », tandis que 33 répondent « non, pas vraiment » ou «non, pas du tout ». Après une formation et 5 mois de pratique, les difficultés à intégrer l'enseignement de la grammaire à la classe de langue semblent persister ; ce qui est peu surprenant si tôt dans la carrière.

12 Les différentes activités grammaticales possibles en classe ne sont pas pratiquées de manière homogène, comme le montre la figure ci-dessous. 


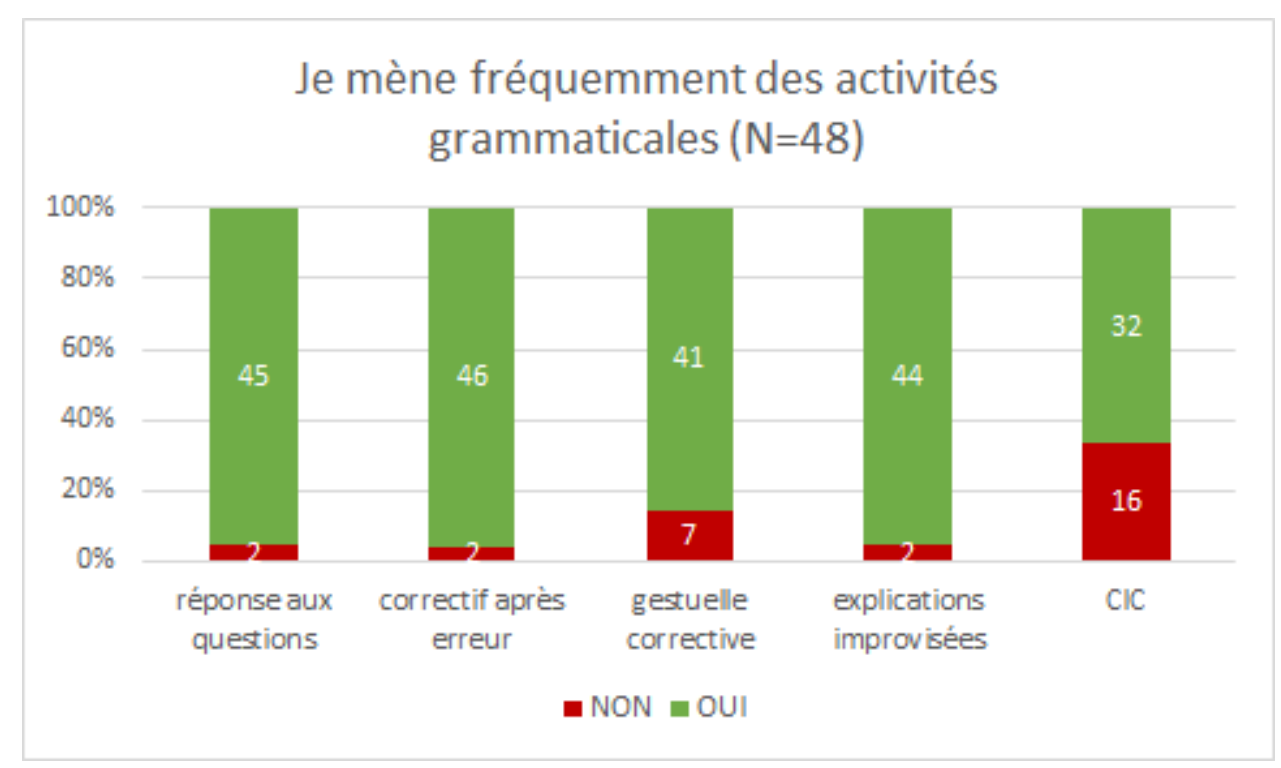

Figure 2 : Questionnaire post-formation à la didactique de la grammaire anglaise. Fréquence des activités grammaticales menées en classe - INSPE de Paris et Sorbonne Nouvelle - Janvier 2018

Les stagiaires répondent fréquemment aux questions grammaticales que les élèves leur posent, ils proposent des correctifs lorsque leurs élèves font des erreurs de grammaire, la plupart ont mis en place une gestuelle pour favoriser l'auto- et l'inter-correction et ils improvisent souvent des explications pour résoudre une difficulté grammaticale imprévue. Cependant, de manière statistiquement significative, ils sont moins nombreux à proposer des activités de Conceptualisation Inductive Complète, alors même que c'est principalement sur cette activité qu'ont porté la formation et l'entraînement. On ne s'étonne pas que les stagiaires éprouvent encore des difficultés à mener des activités grammaticales ou à vrai dire à mener toute forme d'activités si tôt dans leur carrière. Notons cependant, que pour la CIC, ce sont 20 stagiaires sur 47 qui déclarent ne pas savoir mener une CIC correctement.

On peut s'étonner de ce chiffre élevé alors même que la CIC, contrairement aux trois autres pratiques grammaticales analysées ici, n'est pas improvisée ou en réaction à un événement de classe. En effet, le fait de devoir réagir «à chaud» est identifié par les stagiaires eux-mêmes comme un frein majeur à la bonne réalisation de pratiques grammaticales en classe (20 stagiaires sur 48 le mentionnent). Les trois autres raisons fréquemment choisies par les stagiaires sont le manque de temps, leur incompétence supposée et l'anticipation de la réaction de leur tuteur ou inspecteur (29 stagiaires sur 48), comme le montre la figure 3. 


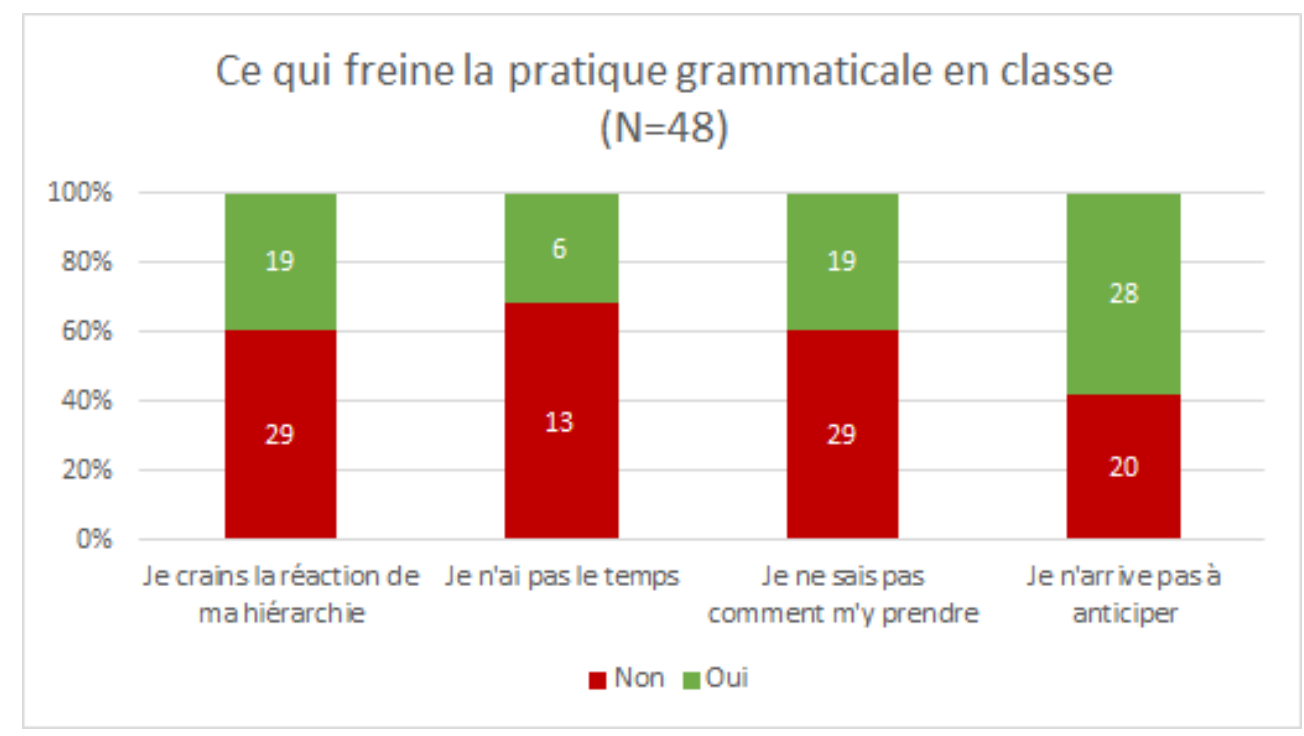

Figure 3 : Questionnaire post-formation à la didactique de la grammaire anglaise. Freins à la pratique grammaticale en classe - INSPE de Paris et Sorbonne Nouvelle - Janvier 2018

Ce dernier point nous semble particulièrement intéressant. Plusieurs commentaires libres précisent que les stagiaires craignent d'être réprimandés s'ils font de la grammaire en classe. Nous avons demandé aux stagiaires ce qu'ils proposaient comme entraînement grammatical en cours, à l'écrit ou à l'oral, mais aussi à la maison. Nous leur avons demandé s'ils proposaient des exercices contextualisés (en rapport avec la séance du point de vue de la thématique), voire si l'entraînement grammatical était intégré à des tâches. Les résultats montrent que la nécessité d'intégrer autant que faire se peut la grammaire à une démarche actionnelle est perçue par 33 stagiaires sur 47 . L'intégration de la pratique grammaticale à des tâches plutôt qu'une pratique décontextualisée est préférée de manière statistiquement significative. De même, 40 stagiaires sur 47 déclarent proposer des exercices contextualisés. Cette préférence pour les exercices contextualisés sur les exercices non contextualisés est également statistiquement significative.

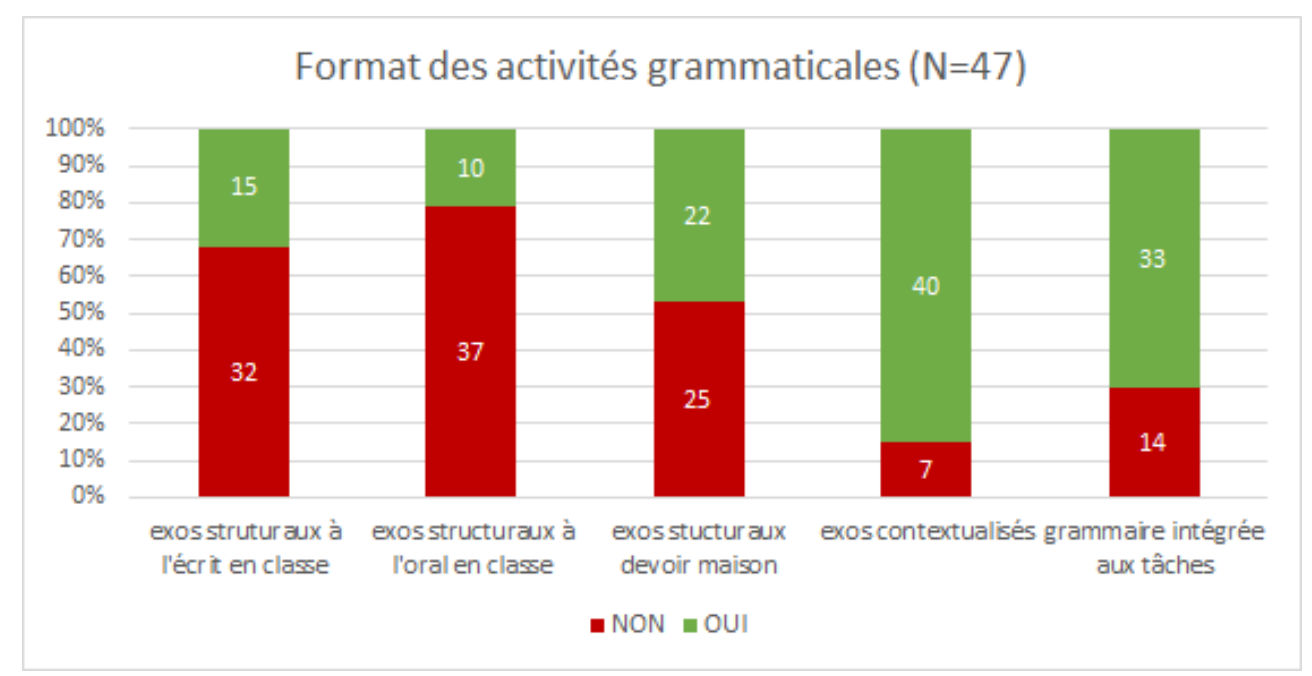

Figure 4 : Questionnaire post-formation à la didactique de la grammaire anglaise. Format des activités grammaticales proposées par les stagiaires - INSPE de Paris et Sorbonne Nouvelle - Janvier 2018 
Cependant, il nous faut remarquer que les exercices structuraux coupés de la démarche actionnelle du cours sont tout de même proposés par de nombreux stagiaires (près de la moitié donnent des exercices structuraux en devoir à la maison) et sont source de questionnement. Le questionnement ne porte cependant pas sur l'efficacité de ces exercices mais sur leur légitimité, comme le montrent les extraits de commentaires libres ci-dessous.

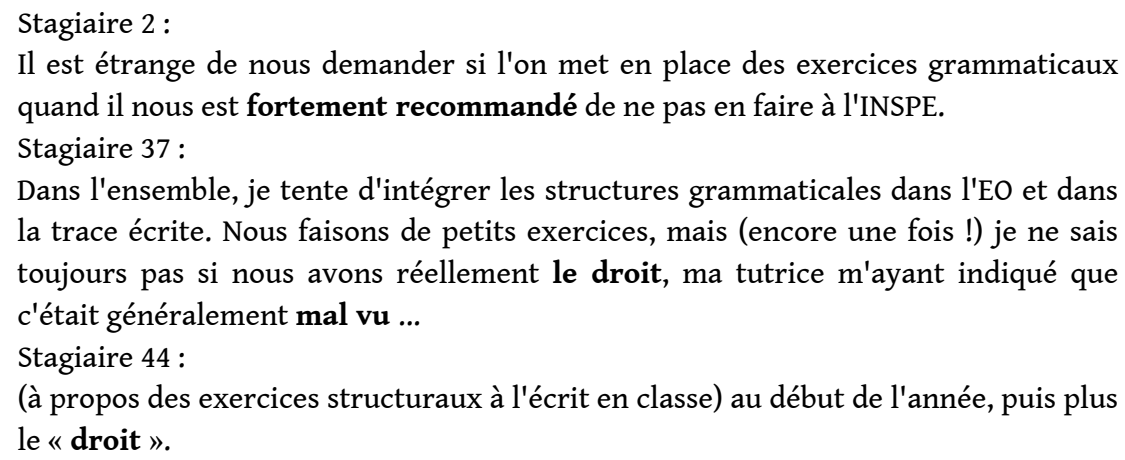

17 D'autres vivent le fait de faire des exercices de grammaire comme une rébellion plus ou moins inconfortable. Mais la question de l'utilité de ces exercices n'est toujours pas posée. Nous reproduisons les commentaires verbatim.

Stagiaire 6:

J'assume de faire des points de grammaire et de faire des exercices structuraux.

Stagiaire 37 :

Je suis assez confuse. On nous prohibe de faire de vrais « points » de grammaire en dehors de la pratique actionnelle. J'en fais quand même mais comment planifier de grands récapitulatifs et/ou exercices si cela nous est aussi fortement non recommandé ?

Il nous semble que de telles interrogations peuvent recevoir plusieurs explications. L'une d'elles peut être que ces stagiaires, lorsqu'ils étaient étudiants, n'ont pas eu l'occasion de s'interroger sur la pertinence de tels exercices et estiment a priori qu'ils aident à progresser dans la maîtrise de la langue parce que c'est le message implicite qui leur a été transmis durant leurs années d'études universitaires. Pourquoi leur aurait-on fait faire des exercices si ceux-ci ne servaient pas à progresser en langue ? Cela est d'autant plus vrai que le moment où les étudiants de licence à la Sorbonne Nouvelle font ou corrigent les exercices est le moment où ils étaient le plus actifs durant les cours de grammaire en licence.

En somme, les stagiaires de M2 MEEF anglais sondés ont une vision assez floue de ce à quoi elle sert. Ils voient le fait de faire des exercices ou non comme une mode assez arbitraire, imposée par la hiérarchie. Enfin, ils trouvent difficile d'intégrer la grammaire à une démarche actionnelle.

\section{Intégration d'éléments agentifs dès la licence}

Pour mieux comprendre les difficultés rencontrées par les stagiaires de M2 MEEF anglais, il nous faut remonter à la formation que la plupart d'entre eux ont reçue en licence LLCER d'anglais. Car si tous les étudiants en licence d'anglais ne deviendront pas enseignants, l'immense majorité des stagiaires M2 MEEF anglais ont été des 
étudiants de licence d'anglais et ont suivi les enseignements frontaux et transmissifs décrits plus haut.

Nous nous sommes donc interrogées sur l'effet de la confrontation entre une approche actionnelle préconisée au collège et au lycée et une pratique frontale et transmissive privilégiée à l'université, toujours en nous appuyant sur le cas de la licence à la Sorbonne Nouvelle.

Certes, les apprenants sont plus âgés. Il est vrai aussi que l'on passe d'un cours de langue pour communiquer dans le secondaire à un cours de grammaire à l'université dont l'objectif, nous l'avons dit plus haut, est davantage de développer une expertise en linguistique que de renforcer une maîtrise de langue souvent considérée (à tort à notre avis, mais c'est une autre question) comme acquise. Mais on peut se demander pourquoi il faudrait dispenser les étudiants de licence d'anglais des apports de la pédagogie par la tâche, en particulier lorsque ce qu'on enseigne est plus abstrait.

Le projet de recherche-action Rénovagram est né en 2015-2016 suite au constat de désintérêt, d'absentéisme et d'échec des étudiants de Licence en grammaire anglaise à la Sorbonne Nouvelle. L'équipe enseignante a souhaité remotiver les étudiants en les rendant plus actifs dans leurs apprentissages. Lapaire, dans un article sur la réception du discours grammatical, rappelle que même si l'enseignant produit un discours savant exact et précis et les analyses les plus fines, il reste encore aux apprenants à apprendre. On ne peut pas faire « comme s'il suffisait d'être plus précis pour être mieux compris, comme si l'intelligence rénovée d'une analyse en faisait nécessairement une proposition plus intelligible pour l'élève ou pour l'étudiant » (Lapaire, 2016).

La grammaire anglaise à l'université ne s'apprend pas plus en écoutant passivement son enseignant que l'anglais pour communiquer ne s'apprend dans le secondaire en écoutant son professeur parler anglais. Nous avons donc procédé à un certain nombre de modifications dans la façon dont était enseigné le cours sur le groupe nominal en $2^{\text {ème }}$ année de licence LLCER.

Mais comment s'y prendre pour proposer une tâche authentique à faire accomplir aux étudiants en classe de linguistique puisqu'il n'y a pas de situation extra-curriculaire où l'on est amené à conduire une analyse linguistique ? Nous avons donc abandonné l'idée d'une approche strictement actionnelle pour favoriser des pratiques pédagogiques impliquantes.

\subsection{Nature des mots lexicaux}

C'est ainsi que dès la première séance sur le groupe nominal, nous avons mis en place des activités mettant les étudiants en action. Plutôt que de leur faire un exposé sur la nature des mots et les critères morphologiques, syntaxiques et sémantiques permettant de les reconnaître, nous leur proposons une activité réflexive en envoyant de petits groupes d'étudiants à l'extérieur de l'université pour effectuer un micro-trottoir d'une quinzaine de minutes. Leur mission est d'interroger trois passants, de leur demander de citer des noms en français puis d'expliquer comment ils savent qu'un mot est un nom. Bien souvent, ceux-ci citent des noms désignant des objets concrets qui les entourent (voiture, poubelle, cigarette ...), plus rarement des notions abstraites, et se justifient par leurs souvenirs scolaires ("Je l'ai appris à l'école») ou sur leur intuition ("Je le sais parce que je le sais »). Ainsi, lors de la mise en commun avec le reste de la classe, un premier critère sémantique est dégagé : un nom peut dénoter une personne, un animal 
ou un objet concret. La réflexion collective permet d'affiner ce critère en faisant produire des exemples divergents.

Incidemment, cette activité liminaire leur indique le format de la participation attendue pour ce cours. Elle ne se limitera pas à écouter, prendre des notes et corriger des exercices. Cependant, nous laissons la possibilité aux étudiants qu'une activité mettrait en difficulté d'opter pour une autre forme d'implication. Par exemple, l'activité alternative au micro-trottoir était un travail sur un corpus contrôlé où les étudiants, en groupe ou seuls, devaient lister les caractéristiques morphologiques et syntaxiques des noms sur de grands posters papier. Les étudiants sondeurs étaient invités à leur retour à confronter les propositions des étudiants restés en classe à leurs collectes.

\subsection{Le système des articles en anglais}

Nous avons étendu cette même démarche inductive à tout un chapitre sur la détermination nominale, abordé lors du premier semestre de la licence 2. Là aussi, plutôt que de décrire les régularités sur les articles en anglais, nous sommes parties d'un corpus contrôlé et nous avons amené les étudiants à formuler les règles par euxmêmes.

Pour ce faire, nous présentons par le biais d'un diaporama une série d'énoncés lacunaires à compléter par un article $(a(n) /$ the ou l'article zéro. Les étudiants répondent grâce à leur boîtier de vote, en se fondant sur leurs connaissances antérieures, acquises au lycée. Après chaque diapositive, nous affichons un histogramme qui présente les réponses des étudiants et indique la réponse correcte. Mais à ce stade, nous ne fournissons pas davantage d'explications. Les énoncés défilent et les étudiants notent les bonnes réponses. Le quiz aboutit à une activité réflexive à mener en pairs invitant les étudiants à formuler des régularités. Les hypothèses sont ensuite vérifiées (en classe entière puis les règles de fonctionnement sont notées. On rejoint ici les phases centrales de la CIC que nous décrivions plus haut.

Par exemple, les étudiants remarquent que les noms propres ne sont pas précédés d'un déterminant, sauf dans quelques cas. Nous les aidons à préciser ces cas pour arriver à une règle comme "Parce que les noms propres sont auto-référentiels, ils ne sont pas précédés d'un déterminant. Ex : Alaska. Cependant, si le noyau du GN est un nom commun, un déterminant est nécessaire, même si le GN désigne un singleton. Ex : the Eiffel Tower. » Nous affinons cette règle en nous appuyant sur des cas chaque fois plus complexes.

\subsection{Structure du groupe nominal}

31 Avant la rénovation du cours, l'étude de la structure du groupe nominal occupait une demi-page sur la brochure qui en contenait cent vingt-cinq. Le passage se terminait par le tableau, reproduit dans la figure 5 , décrivant les différents éléments constitutifs du groupe nominal. 


\begin{tabular}{|c|c|c|c|}
\hline \multicolumn{4}{|c|}{$G \quad R O U C P$} \\
\hline DÉTERMINANT & (MODIFIEUR) & NOM & (MODIFIEUR) \\
\hline An & old & $\operatorname{man}$ & $\begin{array}{l}\text { with a strange accent } \\
\text { groupe prépositionnel }\end{array}$ \\
\hline$\varnothing$ & & People & \\
\hline His & & father & \\
\hline The & very strange & person & $\begin{array}{l}\text { that I told you about } \\
\text { proposition relative }\end{array}$ \\
\hline \multicolumn{4}{|c|}{$\begin{array}{c}\mathrm{He} \\
\text { pronom }\end{array}$} \\
\hline \multicolumn{4}{|c|}{$\begin{array}{l}\text { Mr Smith } \\
\text { Nom propre }\end{array}$} \\
\hline
\end{tabular}

Figure 5 : Tableau récapitulatif sur la structure du groupe nominal en anglais. Support de cours de grammaire anglaise, Licence, deuxième année, avant rénovation : 2014/2015 important de comprendre que les groupes, et plus spécifiquement les groupes nominaux, ont une structure, une hiérarchie qui contredit l'apparente linéarité de la langue? Pour que les étudiants soient actifs dans la compréhension de ce phénomène, nous leur avons proposé des énoncés contenant une ambiguïté syntaxique, tels que :

(1) Look at the dog with one eye

[Regarde le chien avec un œil].

avons demandé aux étudiants de dessiner la double interprétation par groupe de deux. Lors de la mise en commun, nous les avons aidés à représenter la différence de sens. Si c'est le chien qui a un seul œil, le Groupe Prépositionnel " with one eye » fait partie du Groupe Nominal [the dog with one eye], si c'est mon interlocuteur qui doit regarder avec un seul œil, "with one eye » est un Groupe Prépositionnel extérieur au Groupe Nominal : [the dog] [with one eye].

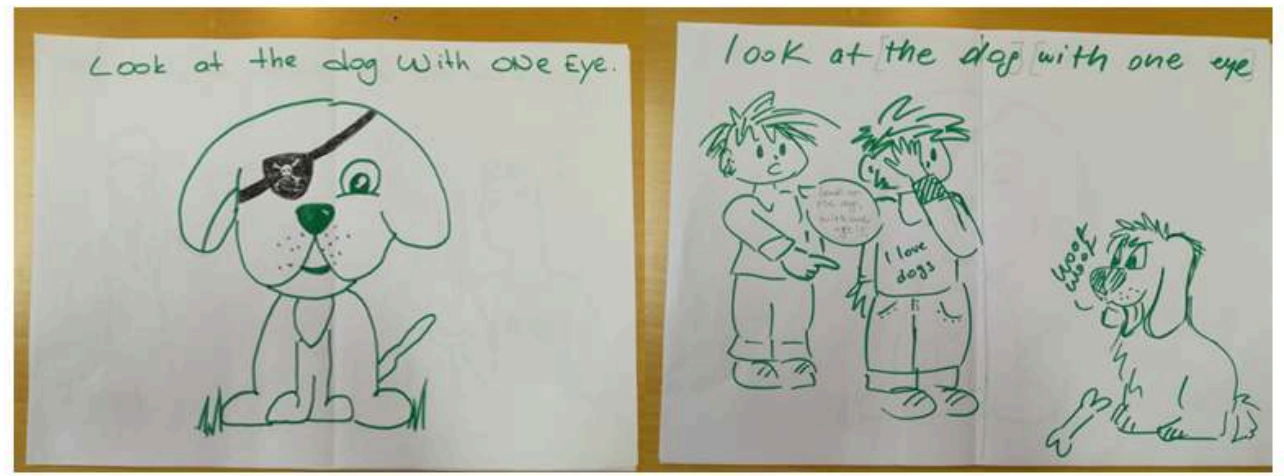

Figure 6 : Dessins d'explicitation par des étudiants de $2^{\text {ème }}$ année de licence d'anglais, Sorbonne Nouvelle, 2018/2019

Nous avons utilisé les boîtiers de vote pour vérifier que les étudiants étaient capables de distinguer une structuration d'un groupe nominal erronée d'une structuration adéquate. Afin de nous assurer qu'ils seraient ensuite capables de décrire par euxmêmes la structure de groupes nominaux, nous avons créé un jeu sérieux (Kapp 2012) 
en détournant le jeu de société «Devine-Tête » que nous avons replacé dans l'univers de la série Game of Thrones très appréciée des étudiants. multiplicité de leurs intelligences, de revenir sur des savoirs et de les assimiler. Grâce au jeu Grammatical Game of Thrones, les étudiants manipulent et jouent avec des concepts aussi abstraits que la post-modification par groupe prépositionnel, et finissent par acquérir des mécanismes qui leur permettent de décrire correctement des groupes grammaticaux.

\section{Perception de ces pratiques pédagogiques}

En 2014/2015, avant d'avoir modifié nos pratiques de classe, nous avions proposé aux étudiants de Licence $2^{\text {ème }}$ année d'évaluer le cours. Nous avons poursuivi cette évaluation jusqu'aujourd'hui en proposant une dizaine de questions identiques tous les ans, quelques questions plus spécifiques à certaines pratiques (boitiers de vote, dessins, présentiel enrichi, badges ...), et un champ de commentaires libres. 2014/2015 nous sert ainsi d'année contrôle, les cohortes, l'équipe enseignante, le programme et le mode de recrutement restant globalement stables.

Chaque année, nous avons proposé la déclaration suivante, volontairement simple " J'aime la grammaire ». Les étudiants pouvaient répondre « oui vraiment », " oui plutôt 
", « non pas vraiment », « non pas du tout ». Notre objectif était de savoir quel pourrait être le niveau de résistance de notre public. La comparaison entre 2014/2015 et 2018/2019 montre une évolution statistiquement significative.

\section{Les étudiant.e.s aiment davantage la matière}

$\left(\chi^{2}(1, N=424)=4,5551 ; p<0,05\right)$

\section{J'aime la grammaire \\ EEE 2014/2015 ( $N=164)$ et 2018/2019 ( $N=260)$}

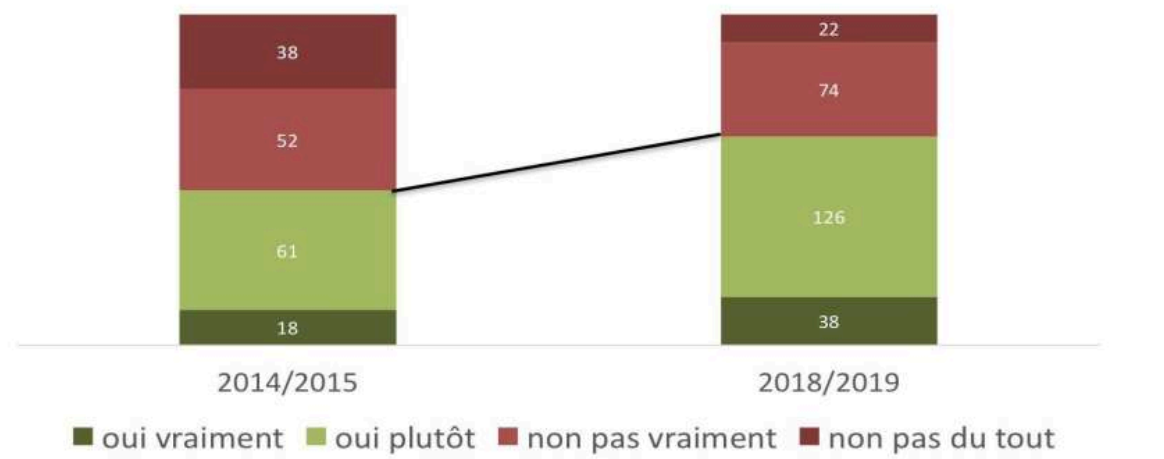

Figure 7 : Évolution du goût des étudiants pour la matière. Enquête EEE auprès de la cohorte d'étudiants de $2^{\text {ème }}$ année, Licence d'anglais, Sorbonne Nouvelle

En 2014/2015, 47\% des étudiants déclaraient aimer la grammaire contre $63 \%$ en 2018/2019. La diminution des étudiants qui répondent " non pas du tout » est également significative. Nous ne pouvons bien entendu pas prouver de relations de cause à effet mais les résultats statistiques pointent au moins en direction d'une corrélation entre l'amélioration de l'expérience d'apprentissage et la modification des pratiques pédagogiques, toutes choses étant égales par ailleurs.

D'autre part, nous avons demandé aux étudiants de 2018/2019 ce qu'ils avaient pensé des pratiques pédagogiques. Face à la déclaration "J'ai aimé participer à un cours innovant », seuls 8 étudiants $(\mathrm{N}=254)$ ont répondu « non pas vraiment » ou « non pas du tout » et 7 n'ont pas vu en quoi le cours était innovant. 


\section{Goût pour cette forme d'enseignement/apprentissage}

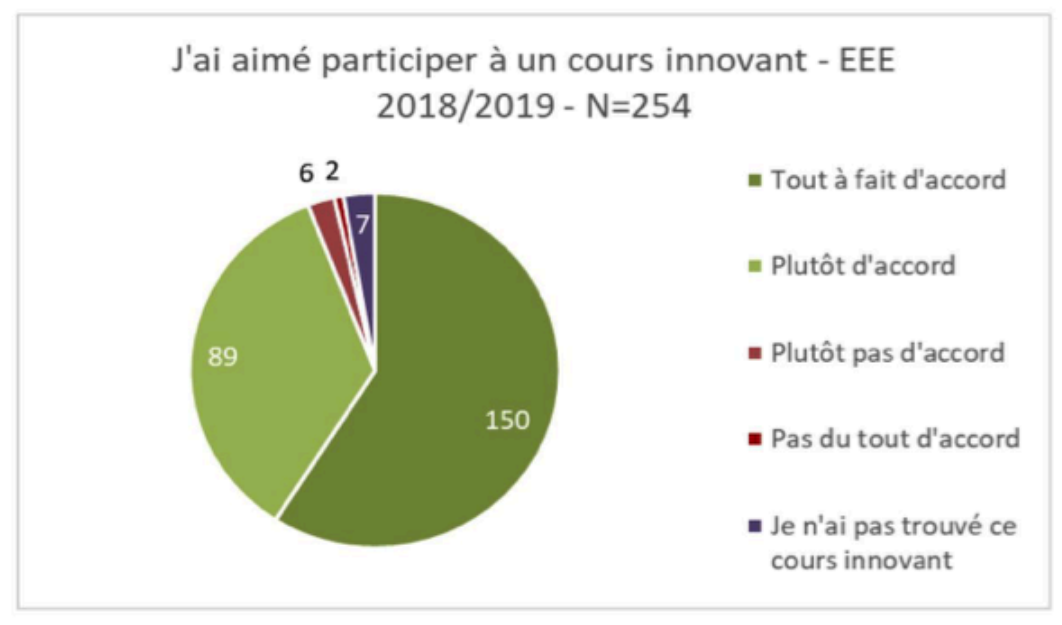

Figure 8 : Goût pour les pratiques pédagogiques impliquantes. Enquête EEE auprès de la cohorte d'étudiants de 2ème année, Licence d'anglais, Sorbonne Nouvelle

En somme, les étudiants de 2018/2019 apprécient majoritairement les approches déployées. Ils estiment également qu'elles contribuent à l'amélioration des apprentissages. Ainsi, en 2018/2019, nous avons interrogé en détail les étudiants sur le jeu grammatical Game of Thrones. $83 \%$ des répondants $(\mathrm{N}=254)$ estiment que le jeu les a aidés à mieux décrire les structures nominales. Il semble important de vérifier que ce ressenti va de pair avec l'effectivité de l'apprentissage. Malheureusement, nous ne savions pas en 2014/2015 que nous nous lancerions dans une recherche-action et nous n'avons pas conservé les productions de nos étudiants. Nous ne pouvons pas non plus nous appuyer sur leurs résultats pour vérifier si la cohorte 2014/2015 a mieux ou moins bien appris que la cohorte 2018/2019 sur laquelle cet article s'appuie. En effet, le format des évaluations a évolué avec la rénovation du cours. De plus, une telle comparaison doit se faire sur des items clairement identifiés et non sur des résultats généraux à une UE. Nous avons cependant mis en place un protocole en 2019/2020 pour vérifier le niveau de maîtrise d'une cohorte équivalente aux cohortes précédentes sur deux compétences visées : repérer le noyau d'un Groupe Nominal et décrire un Groupe Nominal (segmentation et étiquetage). Les résultats préliminaires font apparaître que 93\% de la cohorte de nos L2 ( $\mathrm{N}=218)$ ont acquis la compétence " trouver le noyau » et que $66 \%$ ont acquis la compétence "segmenter et étiqueter le GN ». L'absence de groupe contrôle ne nous permet pas de conclure que notre proposition d'activité impliquante sur la structure du GN est plus efficace qu'une proposition plus frontale. Elle nous permet peut-être de conclure qu'elle est possible et efficace. Peut-être ces pratiques, en favorisant l'implication et la réflexivité, permettent-elles de donner un sens aux enseignements et anticipent-elles ainsi sur ce que nous constations pour nos étudiants-stagiaires de M2 MEEF : une absence d'interrogation sur le bien-fondé de " faire » de la grammaire. 


\section{En guise de conclusion}

La formation des enseignants d'anglais ne commence pas en Master. Elle débute modestement dès le collège, se poursuit au lycée puis sur les bancs de la licence avant de prendre une tournure plus concrète en Master MEEF. Notre pari est que si les démarches pédagogiques perçues par ces futurs enseignants sont cohérentes, impliquantes sinon actionnelles, alors il leur sera plus aisé par la suite, d'intégrer ces démarches à leurs propres pratiques de classe et de comprendre pourquoi ils le font.

Le CECRL et l'approche actionnelle ne bannissent pas l'enseignement de la grammaire, qu'ils placent au contraire au cœur de la compétence communicative (CECRL, 2001 : 115). Nous avons essayé de montrer ici qu'il était possible d'imaginer des tâches permettant d'approfondir cette compétence grammaticale. Cette approche, plus impliquante pour les étudiants, a permis à un certain nombre d'entre eux de (re)trouver du goût pour cette matière et même pour certains de se projeter dans une éventuelle future carrière d'enseignant comme l'indique ce commentaire extrait de l'EEE Licence $2^{2 \grave{m e}}$ année, cohorte 2017/2018, qui nous tiendra lieu de conclusion :

La façon de faire de la grammaire était innovante, agréable et m'a fait changer d'avis sur la matière (je trouvais ça barbant en classe préparatoire ...). C'est inspirant pour mon projet professionnel.

\section{BIBLIOGRAPHIE}

ANDERSON, J., 1983, The architecture of cognition, Harvard University Press, Cambridge.

BERTHIAUME, D. \& REGE, N., 2013, La pédagogie de l'enseignement supérieur : repères théoriques et applications pratiques, Peter Lang, Bern.

CONSEIL DE L'EUROPE, 2001, Cadre Européen Commun de Référence Pour Les Langues - Apprendre, Enseigner, Évaluer, Unité des Politiques Linguistiques, Strasbourg.

JOSSE, H., 2018, « Former les étudiants du CAPES à l'enseignement de la grammaire anglaise », Grammaire? Vous avez dit grammaire? Représentations et pratiques enseignantes, Les Langues Modernes 3.

JOSSE, H., MANOILOV, P., 2019, " Approche grammaticale implicite, explicite, inductive, déductive : quel impact sur l'apprentissage ?», De l'enseignement à l'apprentissage, quelles stratégies ?, Les Langues Modernes 3.

JOSSE, H., RIOU, M., BEAUPOIL-HOURDEL, P., YOUSFI, C., PARIS, J. et al., 2016, « Améliorer l'expérience d'apprentissage en linguistique anglaise : RENOVAGRAM, apprendre en faisant ", Workshop de l'Innovation Pédagogique, Paris, hal-01365312.

JOSSE-DE LA GORCE, H. RIOU, M., BEAUPOIL-HOURDEL, P., BOUQUET-YSOS, C., 2018, «Grammaire, bienveillance et boîtiers de vote : analyse d'une rénovation pédagogique ", Recherche et pratiques pédagogiques en langues de spécialité [En ligne], 37-1, mis en ligne le 04 février 2018, <http://journals.openedition.org.ezproxy.univ-paris3.fr/apliut/5845> 
GRIGGS, P., CAROL, R., BANGE, P., 2002, « La dimension cognitive dans l'apprentissage des langues étrangères ", Revue française de linguistique appliquée, 2-2, p. 25-38.

KAPP, K., 2012, The Gamification of Learning and Instruction: Game-based Methods and Strategies for Training and Education, John Wiley \& Sons, San Francisco, USA.

LAPAIRE, J.R., 2016, « Pour une théorie de la réception du discours grammatical », Anglophonia [en ligne], 22, <http://journals.openedition.org/anglophonia/1051>

SPADA, N., LIGHTBOWN, P., 2008, « Form-Focused Instruction: Isolated or Integrated? » TESOL Quarterly 42-2, p. 181-207.

TALBOT, L., 2012, « Les recherches sur les pratiques enseignantes efficaces », Questions Vives [En ligne] 6-8, mis en ligne le 26 mai 2014, consulté le 13 décembre 2019. <http:// journals.openedition.org/questionsvives/1234 ; DOI : 10.4000/questionsvives.1234>

THERER, J., WILLEMART, C., 1984, « Styles et Stratégies d'enseignement et de formation Approche paradigmatique par vidéo », Education Tribune Libre, Probio Revue 7-1.

\section{RÉSUMÉS}

Les étudiants qui se destinent à passer les concours de recrutement du secondaire bénéficient à la Sorbonne Nouvelle (et sans doute ailleurs) de cours majoritairement frontaux et transmissifs durant toute leur formation initiale, que ce soit en licence LLCER ou en master MEEF Anglais, 1 ère année. Ils sont préparés à passer un concours qui vérifie leurs connaissances en linguistique anglaise et leur capacité à produire des analyses linguistiques, et non leur capacité à l'enseigner dans le secondaire dans le cadre d'une pédagogie actionnelle où l'enseignement de la grammaire reste nécessaire quoique marginal. C'est en $2^{\text {ème }}$ année du Master MEEF anglais, alors que les étudiants, devenus stagiaires, sont déjà en position d'enseignement, que nous leur présentons une méthode pour intégrer le point de grammaire à une séquence actionnelle et leur donnons la possibilité de pratiquer dans le cadre du cours. Nous suggérons ici que l'année de M2 est trop courte pour opérer cette mue nécessaire d'un enseignement frontal et transmissif à une approche pratique et actionnelle. Nous préconisons d'adopter une démarche impliquante tout au long de leur formation universitaire dans la continuité de l'approche actionnelle qu'ils ont connue au lycée. Nous basons notre proposition sur une recherche-action collaborative entamée en 2015 sur des cohortes annuelles de 200 étudiants de deuxième année de licence LLCER anglais $(\mathrm{N}=600)$. En nous appuyant sur nos supports de cours, les enquêtes de satisfaction menées auprès des étudiants à l'issue de chaque semestre, et leurs copies d'examens, nous montrons qu'il est possible et souhaitable d'enseigner la grammaire à des spécialistes dans une perspective que nous qualifions d'impliquante à défaut d'être actionnelle. Cette démarche favorise à la fois l'intégration de savoir expert utile à la réussite des concours d'enseignement mais aussi la préparation, par l'exemple, à un enseignement où les apprenants sont placés en situation d'acteurs et non de récepteurs.

Students who intend to take the recruitment exams to become secondary school teachers are predominantly exposed to frontal and transmissive courses at Sorbonne Nouvelle university (and probably elsewhere) throughout their initial training, during their BA and their first year of MA. They are trained for a competitive exam that checks their knowledge of English linguistics and their ability to produce linguistic analyses, and not their ability to teach grammar in secondary schools in the context of an action-oriented pedagogy where the teaching of grammar is considered as necessary and yet remains marginal. The students have to wait for the 2nd year of their MA, when they have become interns with teaching responsibilities, to be taught a method 
to integrate grammar into an action-based class, and to be given the opportunity to practice that method among their fellow students. Our paper suggests that the $2^{\text {nd }}$ year of Master is too short to make this necessary shift from a frontal transmissive teaching to a practical action-oriented approach. We recommend adopting an engaging approach throughout their university training, in the continuity of the action-oriented approach they experienced as pupils in high school. Our contention is based on a collaborative action-research initiated in 2015 and targeted at annual cohorts of 200 undergraduate second-year English Majors $(\mathrm{N}=600)$. Our data is made up of the course material, satisfaction surveys conducted at the end of each semester, and the students' exam papers. We show that it is both possible and desirable to teach grammar to English Majors using an engaging approach - as we cannot describe our approach as fully action-based. It promotes the learning of expert knowledge that will help the students pass their teaching exam. It also prepares them to teach in classrooms where pupils are meant to be active learners.

INDEX

Mots-clés : Focalisation sur la forme, focalisation sur le sens, conceptualisation inductive complète, démarche actionnelle, pratique pédagogique impliquante.

Keywords : Form-focused Instruction, Meaning-focused Instruction, full inductive conceptualization, task-based language teaching, active pedagogical practice

\section{AUTEURS}

\section{HÉLÈNE JOSSE DE LA GORCE}

Paris-Sorbonne Nouvelle, groupe SESYLIA, EA 4398

\section{CÉLINE THUREL}

Paris-Sorbonne Nouvelle, groupe SESYLIA, EA 4398 